\title{
A mean field analysis of a stochastic model for reservation in car-sharing systems
}

\author{
Christine Fricker ${ }^{*}$ \\ INRIA Paris \\ christine.fricker@inria.fr
}

\author{
Hanene Mohamed ${ }^{\dagger}$ \\ Université Paris Nanterre \\ hmrad@parisnanterre.fr
}

\author{
Cedric Bourdais ${ }^{\ddagger}$ \\ INRIA Paris \\ cedric.bourdais@polytechnique.org
}

\section{INTRODUCTION}

Over the past decade, vehicle-sharing systems have appeared as a new answer to mobility challenges, like reducing congestion, pollution or travel time for numerous cities.

For bike-sharing systems, users make one-way trips. The usage is the following: Users pick a bike at a station if one is available. Otherwise usually they leave the system and take another mode of transportation. After the trip, they return the bike at another station if there is an available space. Otherwise, they have to find a neighboring station to return the bike. In car-sharing systems, users have the ability to reserve, for example the parking space, avoiding the problem of looking for an available space at destination. Again, they can only do this if there are cars and spaces available. The lack of resources, both vehicles and parking slots, is one of the major issues for operators to maintain the reliability of the service (see [6], [1]).

In this paper we analyze a simple homogeneous model for car-sharing systems with one-way trips where users reserve the parking space the moment the car is picked up. As far as we know, it is the first stochastic model of a large-scale vehicle-sharing system with reservation.

Model Description. The system is a set of $N$ stations with capacity $K$ with $M$ cars. Users arrive at each station according to a Poisson process with rate $\lambda$. An arriving user at station $i$ chooses a destination $j$ at random. If there is no car available in station $i$ or if there is no available parking space in station $j$, the user leaves the system. Otherwise, he picks up a car at station $i$ and simultaneously makes a reservation at station $j$. Then, the trip between station $i$ and station $j$ takes an exponentially distributed time with mean $1 / \mu$. At the end of the trip, the user returns his car at station $j$ and leaves the system.

In this paper, we focus on this homogeneous model. But we can extend the result to a heterogeneous model consisting in a finite number of clusters with capacity $K_{i}$ and arrival rate $\lambda_{i}$ for a station in cluster $i$ and probability $p_{i}$ of choosing a destination in cluster $i$, instead of $1 / N$, as in [4].

Large Scale Behavior For this model, the state process of the numbers of cars and reserved parking spaces is an irreducible Markov process on a finite state space with no explicit expression for the unique invariant measure. Thus the aim is to investigate the large scale behavior of the system. This means asymptotics when the number of cars and stations are large together, i.e. that their ratio tends to a con-

MAMA, Boston 2020

Copyright is held by author/owner(s). stant. Our first result (Theorem 1) is the convergence when the system gets large of the distribution of a station state to the distribution of a non linear inhomogeneous Markov process. This distribution is given by a differential equation called Fokker-Planck equation. It is obtained as the limit of the empirical distribution process, as the state process of the model for fixed $N$ has an untractable invariant measure.

Our second main result (Theorem 2) establishes the existence and uniqueness of the equilibrium point of this ODE. The proof uses a monotonicity argument similar as in [3], but here with intricate calculations. We can fully characterize it using probabilistic interpretation.

Our goal is to study the system behavior in terms of large scale stationary proportion of empty and full stations, especially the influence of the fleet size. For the optimal fleet size, we give asymptotics for this quantity in light and heavy traffic. We prove that, in light traffic case, reservation has little impact, unlike the heavy traffic case.

\section{STOCHASTIC MODEL}

\subsection{Empirical Measure Process}

Let us define $\chi=\left\{(k, l) \in \mathbb{N}^{2}, k+l \leq K\right\}$ and let $Y_{k, l}^{N}(t)$ be the proportion of stations with $k$ reserved parking spaces and $l$ cars at time $t$,

$$
Y_{k, l}^{N}(t)=\frac{1}{N} \sum_{i=1}^{N} \mathbf{1}_{\left\{R_{i}^{N}(t)=k, V_{i}^{N}(t)=l\right\}}
$$

where $R_{i}^{N}(t)$ is the number of reserved parking spaces and $V_{i}^{N}(t)$ the number of cars at station $i$ at $t$. Due to homogeneity, $\left(Y^{N}(t)\right)=\left(Y_{k, l}^{N}(t)\right)_{(k, l) \in \chi}$ is a Markov process on

$\mathcal{Y}^{N}=\left\{y=\left(y_{k, l}\right) \in \mathcal{P}(\chi), y_{k, l} \in \mathbb{N} / N, \sum_{(k, l) \in \chi}(k+l) y_{k, l} N=M\right\}$

where $\mathcal{P}(\chi)$ is the set of probability measures on $\chi$. Its transitions from $y$ are the following.

- Cars picked up. A user arrives at a station in state $(k, l)$ at rate $\lambda y_{k, l} N$ if $k>0$. At the same time he reserves in a station in state $\left(k^{\prime}, l^{\prime}\right)$ with probability $y_{k^{\prime}, l^{\prime}}$ if $k^{\prime}+l^{\prime}<K$. Thus, the transition rate is $\lambda y_{k^{\prime}, l^{\prime}} y_{k, l} N$ if $l>0, k^{\prime}+l^{\prime}<K$. Due to arrival, $y_{k, l}$ decreases by $1 / N$ and $y_{k, l-1}$ increases by $1 / N$. Due to reservation, $y_{k^{\prime}, l^{\prime}}$ decreases by $1 / N$ and $y_{k^{\prime}+1, l^{\prime}}$ increases by $1 / N$.

- Cars returned. When a car arrives at its reserved parking space in a station in state $(k, l), y_{k, l}$ decreases by $1 / N$ and $y_{k-1, l+1}$ increases by $1 / N$. The number of 
reserved parking spaces, in stations with state $(k, l)$, is $l N y_{k, l}$. As the trips are exponentially distributed with mean $1 / \mu$, this transition occurs at rate $\mu l N y_{k, l}$.

Jump matrix $Q^{N}$ of process $\left(Y^{N}(t)\right)$ is given by, for $y \in \mathcal{Y}^{N}$,

$$
\left\{\begin{array}{l}
Q^{N}\left(y, y^{\prime}\right)=\lambda y_{k^{\prime}, l^{\prime}} y_{k, l} N \quad \text { if } l>0, k^{\prime}+l^{\prime}<K \\
Q^{N}\left(y, y+\frac{1}{N}\left(\mathbf{e}_{k-1, l+1}-\mathbf{e}_{k, l}\right)\right)=\mu l N y_{k, l}
\end{array}\right.
$$

with $y^{\prime}=y+\frac{1}{N}\left(\mathbf{e}_{k, l-1}-\mathbf{e}_{k, l}+\mathbf{e}_{k^{\prime}+1, l^{\prime}}-\mathbf{e}_{k^{\prime}, l^{\prime}}\right)$ and $\left(\mathbf{e}_{k, l}\right)_{(k, l) \in \chi}$ the vectors of the canonical basis of $\mathbb{R}^{|\chi|}$. As $\left(Y^{N}(t)\right)$ is irreducible and on finite set $\mathcal{Y}^{N}$, it is ergodic with a unique invariant measure. We investigate its limit as $N$ tends to $+\infty$ via a mean-field approach.

\subsection{Dynamical System}

Let us define

$$
\mathcal{Y}=\left\{y \in \mathcal{P}(\chi), \sum_{(k, l) \in \chi}(k+l) y_{k, l}=s\right\} .
$$

TheOrem 1 (MEAN-FIELD CONVERGEnCE THEOREM). For $T>0,\left(Y^{N}(t)\right)_{t \in[0, T]}$ valued in $\mathcal{Y}^{N}$ converges in distribution to $(y(t))_{t \in[0, T]}$ valued in $\mathcal{Y}$, unique solution for a given $y(0)$ of the following $O D E$

$$
\dot{y}(t)=y(t) L_{y(t)}
$$

where $y(t) L_{y(t)}$, product of vector $y(t)$ by jump matrix $L_{y(t)}$, is defined by this detailed form

$$
\begin{gathered}
\dot{y}(t)=\sum_{(k, l) \in \chi} y_{k, l}(t)\left(\lambda\left(1-y_{S}(t)\right)\left(\mathbf{e}_{k, l-1}-\mathbf{e}_{k, l}\right) \mathbf{1}_{l>0}\right. \\
\left.+\lambda\left(1-y_{., 0}(t)\right)\left(\mathbf{e}_{k+1, l}-\mathbf{e}_{k, l}\right) \mathbf{1}_{k+l<K}+\mu l\left(\boldsymbol{e}_{k-1, l+1}-\boldsymbol{e}_{k, l}\right)\right)
\end{gathered}
$$

and $y_{S}(t)=\sum_{k+l=K} y_{k, l}(t)$ and $y_{., 0}(t)=\sum_{k=0}^{K} y_{k, 0}(t)$ are respectively the limiting proportion of saturated stations at $t$ and the limiting proportion of stations with no cars at $t$.

Proof. By standard arguments, for $T>0,\left(Y^{N}(t)\right)_{t \in[0, T]}$ converges in distribution to $(y(t))_{t \in[0, T]}$ unique solution for fixed $y(0)$ of the previous ODE. The first term corresponds to the rate at which users return cars at the reserved parking space, and the second term corresponds to the rate of simultaneous arrival and reservation.

\section{STEADY-STATE BEHAVIOR}

\subsection{Probabilistic Interpretation of the ODE}

The Markov process with generator $L_{y}$ on $\chi$ defined in Theorem 1 can be interpreted as the number of customers in two queues in tandem (see Figure 1): a $M / M / \infty$ queue for reservations, with arrival rate $\lambda\left(1-y_{., 0}\right)$ and service rate $\mu$, and a $M / M / 1$ queue for cars, where customers come from the former queue, with service rate $\lambda\left(1-y_{S}\right)$. Moreover, this system is a loss system as the total number of customers is less than or equal to $K$. An interesting point is the explicit form for the invariant measure $\pi(y)$ of the Markov process.

Indeed, let $\rho_{R}$ be the arrival-to-service rate ratio of the $M / M / \infty$ queue, and $\rho_{V}$ of the $M / M / 1$ queue, defined as

$$
\rho_{R}(y)=\frac{\lambda}{\mu}\left(1-y_{., 0}\right), \quad \rho_{V}(y)=\frac{1-y_{., 0}}{1-y_{S}} .
$$

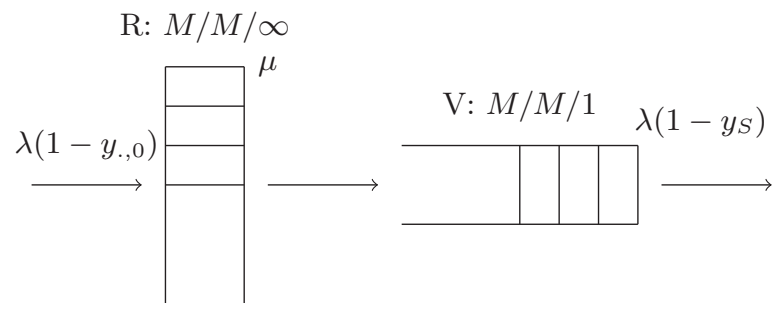

Figure 1: The state of a station as a tandem of two queues with overall capacity $K$.

With these notations, (see [5] for example) the invariant probability measure $\pi(y)$ associated to $L_{y}$ has product form given by, with a slight abuse of notations, for $(k, l) \in \chi$,

$$
\pi_{k, l}\left(\rho_{R}(y), \rho_{V}(y)\right)=\frac{1}{Z\left(\rho_{R}(y), \rho_{V}(y)\right)} \frac{\rho_{R}(y)^{l}}{l !} \rho_{V}(y)^{k}
$$

where $Z\left(\rho_{R}(y), \rho_{V}(y)\right)$ is a normalization constant. In the following, we will use both $\pi\left(\rho_{R}(y), \rho_{V}(y)\right)$ and $\pi(y)$. Since $\pi(y) \in \mathcal{P}(\mathcal{Y})$, we also have

$$
s=\sum_{(k, l) \in \chi}(k+l) \pi_{k, l}(y) .
$$

It can be rewritten $s=\mathbb{E}(V+R)$ where $(R, V)$ is a random variable with distribution $\pi(y)$.

For the steady-state behavior of the model, we are interested by the equilibrium points $\bar{y}$ of the ODE (1). Such equilibrium points $\bar{y}$ satisfies

$$
\pi\left(\rho_{R}(y), \rho_{V}(y)\right)=y
$$

and equations (2) and (4). Thus, this is equivalent to find couples $\left(\rho_{R}, \rho_{V}\right)$ such that $\bar{y}=\pi\left(\rho_{R}, \rho_{V}\right)$, satisfying

$$
\begin{aligned}
\rho_{R} & =\frac{\lambda}{\mu}\left(1-\pi_{., 0}\left(\rho_{R}, \rho_{V}\right)\right), \rho_{V}=\frac{1-\pi_{., 0}\left(\rho_{R}, \rho_{V}\right)}{1-\pi_{S}\left(\rho_{R}, \rho_{V}\right)}(6) \\
s & =\mathbb{E}(R+V)
\end{aligned}
$$

where $(R, V)$ is a random variable with distribution $\pi\left(\rho_{R}, \rho_{V}\right)$. Hence, the question of finding a measure on $\chi$ is reduced to find a couple of real numbers $\left(\rho_{R}, \rho_{V}\right)$.

\subsection{Existence and Uniqueness of the Equilib- rium Point}

Our second main result is the existence, uniqueness and characterization of the equilibrium point.

TheOREM 2 (A UNIQUE EQUILIBRIUM POINT). There is a unique equilibrium point $\bar{y}$ for the solution of ODE (1) given by $\bar{y}=\pi\left(\rho_{V}, \rho_{R}\right)$ where $\pi$ is the invariant measure of a tandem of two queues with total capacity $K$ and respective rates $\rho_{R}$ and $\rho_{V}$ such that $\left(\rho_{V}, \rho_{R}\right)$ is the unique solution of the first equation of (6) and equation (7).

Proof. We present a sketch of the proof (see [2, Theorem $2]$ for a full proof). Recall that finding $\bar{y}$ is equivalent to finding $\left(\rho_{R}, \rho_{V}\right)$ solution of equations (6) and (7). First, the second equation in (6) is true for any $\left(\rho_{R}, \rho_{V}\right)$. Thus $(6)$ amounts to its first equation. Second, this first equation of (6) is an implicit equation in $\rho_{R}$ and $\rho_{V}$. It gives, by implicit function theorem with combinatorial arguments, a diffeomorphism $\phi:\left[0, \lambda / \mu\left[\rightarrow\left[0,+\infty\left[\right.\right.\right.\right.$ such that $\rho_{V}=\phi\left(\rho_{R}\right)$. 
Moreover we prove that $\phi$ is strictly increasing. The third step consists in a combinatorial proof of the monotonicity of the right-hand side of equation (7) as a function of $\rho_{V}$ and $\rho_{R}$. Using the continuity and monotonicity of $\phi$ related $\rho_{R}$ and $\rho_{V}$, we conclude to the monotonicity of the righthand side of equation (7) as a function of $\rho_{R}$. It gives the existence and uniqueness of a $\rho_{R}$ thus of $\left(\rho_{R}, \rho_{V}\right)$, solution of (6) and (7). It ends the proof.

\section{SYSTEM PERFORMANCE}

The aim is to give insight into the optimal behavior for the performance metric. The influence of the fleet size parameter $s$ is investigated. In [2] the authors discuss different performance metrics. Here we focus on the proportion of empty and full stations called problematic. Its asymptotics as the system and time gets large is a function of $\bar{y}$.

Definition 1 (Problematic Stations). Let $\bar{y}$ be the unique equilibrium point of ODE (1). The stations with either no car or no parking space available are called problematic. The limiting stationary proportion $P_{b}$ of problematic stations is given by

$$
P_{b}=\mathbb{P}(V=0 \text { or } V+R=K)=\bar{y}_{., 0}+\bar{y}_{S}-\bar{y}_{0, K}
$$

where $(V, R)$ is a random variable with distribution $\bar{y}$.

\subsection{Influence of the fleet size}

All functions of $\left(\rho_{R}, \rho_{V}\right)$ can be expressed on $\rho_{V}$ only, like $\bar{y}\left(\rho_{V}\right), P_{b}\left(\rho_{V}\right)$ and $s\left(\rho_{V}\right)$. Indeed by first equation in (6), for a fixed $\rho_{V} \in\left[0,+\infty\left[, \rho_{R}=\phi^{-1}\left(\rho_{V}\right)\right.\right.$. Then we get $\bar{y}=\pi\left(\rho_{R}, \rho_{V}\right)$ by (3) and $s\left(\rho_{V}\right)$ by (4). Thus, with abuse of notation, the proportion of problematic stations, as a function of the fleet size, is given by the parametric curve

$$
\rho_{V} \mapsto\left(s\left(\rho_{V}\right), P_{b}\left(\rho_{V}\right)\right) .
$$

This curve gives the influence of the fleet size on the behavior of the system. It is numerically plotted in Figure 2 and compared to the same curve for bike sharing systems studied in [3] using the same performance metric.

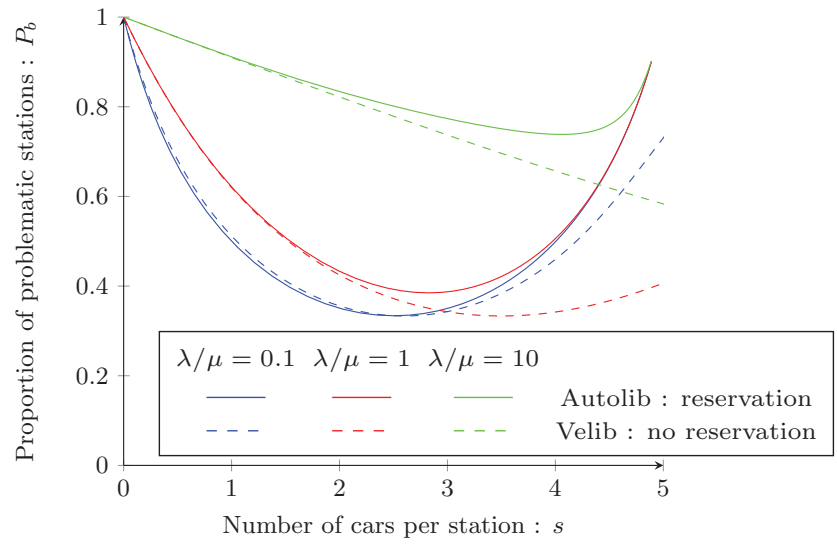

Figure 2: Asymptotic stationary proportion of empty and full stations as a function of the fleet size $s$ for station capacity $K=5$ (parametric curve).

Figure 2 shows a minimum for $P_{b}$ as a function of $s$. We can characterize it by this result.

\begin{tabular}{|c|c|r|}
\hline & light traffic: $\lambda / \mu \rightarrow 0$ & heavy traffic: $\lambda / \mu \rightarrow+\infty$ \\
\hline$s^{*}$ & $\frac{K}{2}+\frac{K^{2}}{2(K+1)^{2}} \frac{\lambda}{\mu}$ & $K-\sqrt{\frac{K}{\lambda / \mu}}+\frac{2-K}{\lambda / \mu}$ \\
\hline$P_{b}^{*}$ & $\frac{2}{K+1}+\frac{2 K}{(K+1)^{3}} \frac{\lambda}{\mu}$ & $1-\frac{K-1}{\lambda / \mu}+\frac{2(K-1)}{\sqrt{K}}\left(\frac{\lambda}{\mu}\right)^{-3 / 2}$ \\
\hline
\end{tabular}

Table 1: First terms for expansions in $\lambda / \mu$ at 0 (light traffic) and $+\infty$ (heavy traffic) of the optimal proportion of problematic stations $P_{b}^{*}$ for $s^{*}$ cars per station.

Proposition 1. $P_{b}$ has an extremum $P_{b} *$ for $\rho_{V}=1$.

Proof. The following interesting property of symmetry can easily be checked. For $\rho_{V}>0, \phi^{-1}\left(1 / \rho_{V}\right)=\phi^{-1}\left(\rho_{V}\right) / \rho_{V}$ and $P_{b}\left(1 / \rho_{V}\right)=P_{b}\left(\rho_{V}\right)$. Thus $P_{b}^{\prime}\left(\rho_{V}\right)=-P_{b}^{\prime}\left(1 / \rho_{V}\right) / \rho_{V}^{2}$. Therefore, $P_{b}^{\prime}(1)=-P_{b}^{\prime}(1)=0$.

Uniqueness of the minimum should come from the convexity of the parametric curve $\rho_{V} \mapsto\left(s\left(\rho_{V}\right), P_{b}\left(\rho_{V}\right)\right)$. Nevertheless this convexity remains to prove, the implicit relation between $\rho_{V}$ and $\rho_{R}$ making calculations tedious. This fact is similar to the result in [3, Theorem 1] for bike-sharing systems, that the minimum is reached for $\rho_{V}=1$.

\subsection{Optimal fleet size}

We can compute asymptotics for $P_{b}$ and $s$ for any $\rho_{V}$ in two cases: light $(\lambda / \mu \rightarrow 0)$ and heavy $(\lambda / \mu \rightarrow+\infty)$ traffic (see [2] for details). Then taking $\rho_{V}=1$ gives expansions in $\lambda / \mu$ for the optimal value $P_{b}^{*}$ for $s^{*}$. The first terms of the expansions are presented in Table 1 .

For light traffic, the system has the same optimal performance at first order in $\lambda / \mu$ as the homogeneous bikesharing model, where it is $2 /(K+1)$ for each $\lambda / \mu$ (see [3, Theorem 1]). Indeed, intuitively, reservation does not induce congestion in light traffic case. This result can be observed on Figure 2 where curves for the bike-sharing and car-sharing systems are quite close at optimum only for $\lambda / \mu=0.1$. In case of heavy traffic, $P_{b}^{*}$ tends to 1 quicker compared to the convergence of $s^{*}$ to $K$.

\section{REFERENCES}

[1] A. Carlier, A. Munier-Kordon, and W. Klaudel. Mathematical model for the study of relocation strategies in one-way carsharing systems.

Transportation Research Procedia, 10:374-383, 2015.

[2] C. Fricker and C. Bourdais. A stochastic model for car-sharing systems. arXiv preprint arXiv:1504.03844, 2015.

[3] C. Fricker and N. Gast. Incentives and redistribution in homogeneous bike-sharing systems with stations of finite capacity. Euro journal on transportation and logistics, 5(3):261-291, 2016.

[4] C. Fricker, N. Gast, and H. Mohamed. Mean field analysis for inhomogeneous bike sharing systems. Discrete Mathematics \& Theoretical Computer Science, pages 365-376, 2012.

[5] P. Robert. Stochastic networks and queues, volume 52. Springer Science \& Business Media, 2013.

[6] S. Schmöller, S. Weikl, J. Müller, and K. Bogenberger. Empirical analysis of free-floating carsharing usage: The munich and berlin case. Transportation Research Part C: Emerging Technologies, 56:34-51, 2015. 